489 (1959).

${ }^{8} \mathrm{~V}$. K. Wong Ph. D. thesis, Berkeley, 1966 (unpublished), p. 135.

${ }^{9}$ S. T. Beliaev, Zh. Eksperim. i Teor. Fiz. 34, 433 (1958) [English transl.: Soviet Phys. - JETP ㄱ, 299 (1958)].

${ }^{10}$ K. A. Brueckner and K. Sawada, Phys. Rev. $\underline{106}$,
1117 (1957).

${ }^{11}$ J. Gavoret and P. Nozières, Ann. Phys. (N. Y.) 28 , 349 (1964).

${ }^{12}$ K. Huang and A. Klein, Ann. Phys. (N. Y.) $\underline{30}, 203$ (1964).

${ }^{13}$ P. J. Bendt, R. D. Cowan, and J. L. Yarnell, Phys. Rev. 113, 1386 (1959).

\title{
Third Sound and the Onset of Superfluidity in Unsaturated Helium Films
}

\author{
David Goodstein \\ Department of Physics, California Institute of Technology, Pasadena, California 91109
}

(Received 12 March 1969)

\begin{abstract}
A simple model, commonly used for describing the behavior of the superfluid film is examined, and it is found to become spontaneously unstable when the partial molar entropy falls below a certain finite value for each temperature and film thickness. The instability is proposed as the cause of the onset curve for superfluidity in unsaturated films, and comparisons with experiment are made. In addition, the use of thermodynamic identities in the model leads to a new and more accurate expression for the velocity of third sound in terms of other measurable quantities.
\end{abstract}

\section{INTRODUCTION}

It is well known that liquid $\mathrm{He}^{4}$ under its own vapor pressure undergoes a phase transition at $T_{\lambda} \cong 2.17^{\circ} \mathrm{K}$, below which temperature its properties are unusual enough to merit the term superfluid. It is also known that superfluidity exists in very thin films of helium (down to just a few atomic layers) but the nature of the transition to superfluidity in thin films is obscure. In bulk liquid $\mathrm{He}^{4}$, the transition to superfluidity is accompanied by a logarithmic infinity in the heat capacity. In very thin films the heat capacity has a broad maximum at temperatures slightly lower than $T_{\lambda}$, but the onset of superflow occurs at temperatures considerably lower than the heat-capacity maximum.

If the equilibrium gas pressure, $P$, above a helium film is less than the liquid vapor pressure, $P_{0}$, the film is said to be unsaturated. The thickness of the film, $d$, or amount adsorbed per unit area, $N$, is a definite function of $P$ and $T$ for any given substrate. At each temperature below $T_{\lambda}$ the superflow properties of the film are found to vanish if $P / P_{0}$ drops below some value. A plot of this value of $P / P_{0}$ against temperature is given in Fig. 1. Superflow is observed in the film at all lower temperatures and higher pressures. We shall refer to this curve as the onset curve.

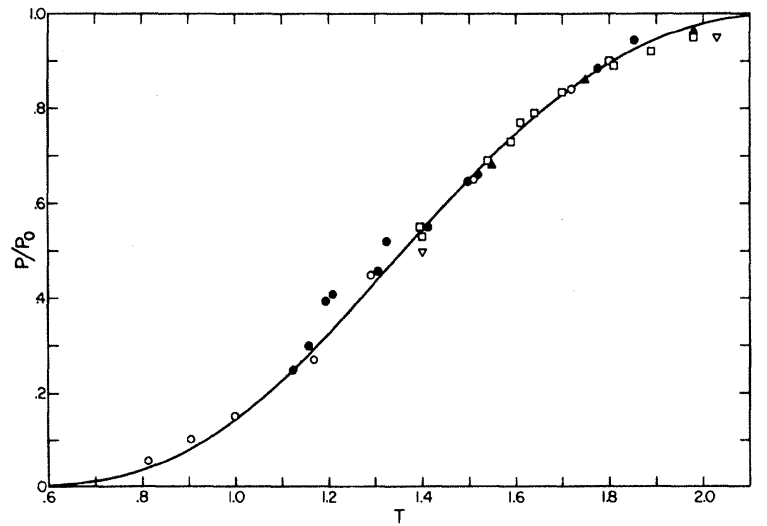

FIG. 1. The curve for the onset of superflow in unsaturated films: $T$ in ${ }^{\circ} \mathrm{K}$ versus $P / P_{0} \quad \nabla$ Long and Meyer, Phys. Rev. 79, 1031 (1950), mass transport; $\square$ Brewer and Mendelssohn, Proc. Roy. Soc. (London) A260, 1 (1961), heat flow; $\bigcirc$ Fokkens, Taconis, and DeBruyn Ouboter, Physica 32, 2129 (1966), heat flow; - Rudnick et al., (Ref, 3), third sound; $\Delta$ Henkel, Kukichi, and Reppy (Ref. 4), persistent currents. In the experiments by Long and Meyer, the film flowed under the influence of a pressure gradient. In some cases (the points shown) agreement with this curve was found. In others superflow all the way up to $T_{\lambda}$ was detected even in very thin film. The discrepancy between these latter results and all other measurements has never been understood. 
Various detection techniques on assorted substrates give good agreement for the onset curve. Most of the results plotted in Fig. 1 are obtained by three techniques: (1) A heat pipe is set up in which superflow through an unsaturated film replenishes helium evaporated at a heater. The thermal conductivity decreases sharply when superflow stops. (2) Persistent currents in the unsaturated film are measured by the superfluid gyroscope technique. The persistent current ceases when there is no superflow. (3) A superfluid wave motion, called third sound, is detected in the unsaturated film. No third sound is detected without superflow.

The existence of third sound was predicted by Atkins, ${ }^{1}$ who developed a model that emphasized the analogy between these superfluid waves and shallow water waves. In the case of third sound, the restoring force was the van der Waals force $f$ due to the underlying substrate, instead of gravity. He pictured a film in which the only flow was that of the superfluid component parallel to the surface, the normal component being clamped by viscosity. This superflow was driven by the gradient of the film's chemical potential,

$$
\stackrel{\circ}{v}_{S}=S_{v} \nabla T-\rho^{-1} \nabla P,
$$

where $S_{v}$ is the entropy per unit volume, $\rho$ the density and $v_{s}$ the superflow velocity. The restoring force $f$ enters by letting the pressure consist of the gas pressure plus an effective pressure $\rho f d$ due to the substrate. The equation for mass conservation took into account evaporation and condensation as well as superflow, and a third equation represented heat flow by evaporation and condensation only, since the superflow carried no entropy. Atkins found that these three equations had wave-like solutions for perturbations in $T$, $d$, and $v_{S}$, with a phase velocity given by

$$
v_{3}^{2} \cong\left(\rho_{s} / \rho\right) f d,
$$

where $\rho_{S} / \rho$ is the superfluid fraction. These waves are called third sound.

Everitt et al. ${ }^{2}$ have detected third sound optically in nearly saturated films. Rudnick and his co-workers ${ }^{3}$ have studied third sound in unsaturated films by detecting the associated temperature wave. It is in these latter experiments that the onset discussed above has been detected by means of third sound.

In both measurements of third sound the data have been interpreted by relating $f d$ in Eq. (1) to the difference in chemical potential between the film and the liquid. In Sec. II we shall rederive Atkins' equations, eliminating $f$ entirely by using the appropriate chemical potential directly in the equation for $v_{S}$.
Rudnick et $a l^{\prime}{ }^{3}$ have concluded from their thirdsound studies that $\rho_{S} / \rho$ does not vanish at the onset curve. Instead it reaches a definite nonzero fraction of the bulk value. Henkel et al. ${ }^{4}$ have reached a similar conclusion based on the persistent current measurements. Goodstein and Elgin $^{5}$ (hereafter referred to as I) have shown that $\rho_{s} / \rho$ is not discontinuous at the onset. It follows, therefore that $\rho_{S} / \rho$ remains finite in the region where no superflow is detected.

Brewer et al. ${ }^{6}$ had earlier proposed a model which predicted a finite $\rho_{s} / \rho$ in this region. They suggested the existence of special excitations, associated with the free surface, which served to stop the superflow. This was to account for the observation that in very fine pores filled with helium, the heat capacity behaved in a way similar to that of thin films (a broad maximum at slightly depressed temperatures) but unlike the film case, in filled pores the onset of superflow concided with the heat-capacity maximum. Since the essential difference seemed to be the presence of a free surface in the film, Brewer et al. proposed a mechanism associated with this free surface.

The possibility of an alternative explanation was pointed to in I. There it was shown that in films of thickness about five atomic layers, in which the onset occurs at about $1.5^{\circ} \mathrm{K}$, the onset is occasioned by a decrease in the partial molar entropy, $\bar{S}$, to a value close to zero. Using Eq. (6) below, it was pointed out that this decrease could account for the disappearance of thermally detected flow, such as third sound.

The partial molar entropy is the derivative of the total entropy with respect to the number of moles present [see Eq. (3) below] and in most bulk phases is identical to the molar entropy。 However, in thin films the molar entropy depends on the thickness of the film, and thus differs from the partial molar entropy. The dependence of entropy on thickness was not taken into account by Atkins, ${ }^{1}$ since he was dealing with nearly saturated films, but its importance for the unsaturated case has been pointed out by Bergmann, ${ }^{7}$ and is included in the reformulation of Atkins' equations in Sec. II below.

In most ordinary substances (e.g., nitrogen, argon) the entropy of a thin film is lower than that of the bulk liquid at the same temperature, so that the molar entropy increases with increasing thickness, and the partial molar entropy is greater than the molar entropy. ${ }^{8}$ However, in the case of helium, the bulk liquid state is highly ordered and the film somewhat less so. The result is that the film generally has a higher molar entropy than the liquid ${ }^{9}$ and the partial molar entropy can be small, or even, in principle, negative.

Suppose at some point the partial molar entropy becomes equal to zero. The meaning of this 
statement is that a change in the amount of helium per unit area in the film will not change the total entropy per unit area in the film. Thus if there is a local change in temperature, heat cannot be conserved by a local inflow (or outflow) of helium. Temperature thus appears to be decoupled from flow. This was the argument used in I.

It is shown below on the basis of the reformulated equations of motion of the film, that if $\bar{S}$ falls below some finite positive value at any temperature, the film becomes spontaneously unstable.

In Sec. II we reformulate Atkins' model of the film, and show that the resulting equations have wave solutions representing third sound and also, under certain conditions, the instability. In addition, the new expression for the velocity of third sound is compared to previous usage. In Sec. III the instability is discussed, and the proposition that it accounts for the onset curve is considered.

\section{THE EQUATIONS OF THE FILM}

Our general formulation of the model of the film follows closely that of Atkins', ${ }^{1}$ differing in only the following 3 ways: (1) Atkins focuses on the interplay between $T, v_{s}$, and $d$, the thickness of the film. We shall use, instead of $d, N$, the amount adsorbed per unit area (in moles per square centimeter)。 $N$ is a more easily measured quantity than $d$. The two are approximately proportional, although the relationship between them depends on the compression of the film due to van der Waals forces. For convenience and clarity we shall continue to refer in qualitative discussions to the thickness of the film since the qualitative behavior of $d$ and $N$ are the same. (2) We shall take into account the fact that the molar entropy of the film, $S$, depends on the film thickness as well as the temperature, $S=S(T, N)$. The dependence of $S$ on $N$ is not significant in the nearly saturated films considered by Atkins, and so he ignored it. (3) We shall assume that if the film is locally perturbed in $T$ and $N$, it is nevertheless locally in internal equilibrium. Thus if the chemical potential of an equilibrium film is some function $\mu_{f}(T, N)$, then when the variables have locally become $T^{\prime}$ and $N^{\prime}$, the chemical potential locally is $\mu_{f}\left(T^{\prime}, N^{\prime}\right)$ 。 Since at equilibrium $\mu_{f}=\mu_{g}$, where $\mu_{g}$ is the chemical potential of the gas above the film, we can represent perturbations in $\mu_{f}$ as equal to changes in the functional form of $\mu_{g \cdot}$ Thus we write, $d \mu_{f}=-S_{g} d T+V_{g} d P$, where $S_{g}$ is the molar entropy and $V_{g}$ is the molar volume of the gas. This is not to imply that the gas is perturbed in this way, nor that the film is locally in equilibrium with the gas; the assumption made about the state of the gas is discussed below.

With Atkins, we assume the normal fluid is clamped by viscosity, ignore motion perpendicular to the substrate, and consider disturbances whose wavelengths are long compared to the thickness of the film. We consider the superfluid velocity, $v_{s}$, to be small, and ignore all terms beyond first order in the disturbances, $v_{s}, \Delta T$, and $\Delta N$. (a steady-state background superflow has no effect on these considerations).

With this model we have, for one-dimensional flow in the $x$ direction, writing $M$ for the atomic mass,

$$
\begin{aligned}
M \frac{\partial v_{s}}{\partial t} & =-\frac{\partial \mu_{f}}{\partial x}=S_{g} \frac{\partial T}{\partial x}-V_{g} \frac{\partial P}{\partial x} \\
& =S_{g} \frac{\partial T}{\partial x}-V_{g}\left[\left(\frac{\partial P}{\partial T}\right)_{N} \frac{\partial T}{\partial x}+\left(\frac{\partial P}{\partial N}\right)_{T} \frac{\partial N}{\partial x}\right] \\
& =\left[S_{g}-V_{g}\left(\frac{\partial P}{\partial T}\right)_{N}\right] \frac{\partial T}{\partial x}-V_{g}\left(\frac{\partial P}{\partial N}\right)_{T} \frac{\partial N}{\partial x} \\
M-\frac{\partial v_{s}}{\partial t} & =S \frac{\partial T}{\partial x}-\alpha \frac{\partial N}{\partial x}
\end{aligned}
$$

where $\alpha=V_{g}(\partial P / \partial N)_{T}$ is a quantity measured in adsorption isotherms $[P(N)$ at constant $T]$, and

$$
\bar{S}=\left(\frac{\partial(N S)}{\partial N}\right)_{T}
$$

$\bar{S}$ is called the partial molar entropy. Since $N S$ is the total entropy per unit area, $\bar{S}$ is just the change in entropy per unit area when $N$ changes. In arriving at $\mathrm{Eq}$. (2) we have used the thermodynamic identity ${ }^{9}, 10$

$$
S_{g}-\bar{S}=V_{g}\left(\frac{\partial P}{\partial T}\right)_{N}
$$

As Eq. (4) makes clear, $\bar{S}$ is a quantity that may be deduced from adsorption isosteres $[P(T)$ at constant $N]$. The quantity $q_{s t}=T\left(S_{g}-\bar{S}\right)$ is sometimes called the isosteric heat of adsorption. ${ }^{10}$

The local rate of evaporation from the film is given by

$$
\left(\frac{\partial N}{\partial t}\right)_{\mathrm{ev}}=\gamma\left(\frac{1}{2 \pi M R T}\right)^{1 / 2}\left(\frac{\partial P}{\partial T}\right)_{N} \Delta T=K \Delta T
$$

where $\Delta T$ is the difference between the local and average temperatures, and $\gamma \approx 1$. Aside from using $(\partial P / \partial T)_{N}$ in place of $d P_{0} / d T$ in order to apply to unsaturated films, Eq. (5) is the same as that used by Atkins. The underlying assumption is that aside from the temperature term, the chemical potential of the gas is locally the same 
as that of the perturbed film. Temperature terms aside, the surface of the film suffers a change in chemical potential when the thickness varies, due to its change in position in the van der Waals field. The gas at the interface, however, suffers the same change, and we assume it is unable to adjust its density before the wave has been restored. Thus the interface is assumed to be an isobaric surface in the gas, but not a surface of constant chemical potential, the van der Waals potential being lower for gas at the interface of a thin spot than a thick spot, just as it is for the film. Essentially, we are assuming that the gas does not respond to changes in potential as fast as does the film, the justification being that while the driving forces are the same, the film is in superflow and the gas is not. ${ }^{11}$ Using Eq. (4) and the assumption that no entropy flows in the film we get

$$
\frac{\partial}{\partial t}(N S)+S_{g}\left(\frac{\partial N}{\partial t}\right)_{\mathrm{ev}}=0 .
$$

Recalling that $S=S(T, N)$ and using Eq. (5), we have

$$
\bar{S} \frac{\partial N}{\partial t}+N \frac{\partial S}{\partial t}=-S_{g} K \Delta T .
$$

We now write $(\partial S / \partial t)_{N}=\left(C_{N} / T\right)(\partial T / \partial t)\left(C_{N}\right.$ is the specific heat) and get

$$
\bar{S} \frac{\partial N}{\partial t}+\frac{N C_{N}}{T} \frac{\partial T}{\partial t}=-S_{g} K \Delta T .
$$

Conservation of mass gives us

$$
\frac{\partial N}{\partial t}+\frac{\partial}{\partial x}\left(N \frac{\rho_{s}}{\rho} v_{s}\right)+\left(\frac{\partial N}{\partial t}\right)_{\mathrm{ev}}=0
$$

or finally,

$$
\frac{\partial N}{\partial t}+\frac{\partial}{\partial x}\left(N \frac{\rho_{s}}{\rho} v_{s}\right)=-K \Delta T .
$$

Equations (2), (6) and (7) together constitute the equations of motion of our model of the film.

Formally, these equations are very similar to those arrived at by Atkins. ${ }^{1}$ They differ only in that $d$ has been replaced by $N, S$ has been replaced by $\bar{S}$, and terms involving $f$ are replaced by terms involving the slopes of adsorption isotherms. ${ }^{12}$

To put the equations into an easy form for manipulation, it is convenient to make the following set of transformations;

$$
v_{3}^{2}=\frac{\rho_{s}}{\rho} \frac{N}{M} V_{g}\left(\frac{\partial P}{\partial N}\right)_{T}=\frac{\rho_{s}}{\rho} \frac{N}{M} \alpha
$$

$$
\begin{aligned}
& \omega_{0}=K T S_{g} / N C_{N}, \quad \bar{\omega}=K T \bar{S} / N C_{N}, \\
& \omega_{0}{ }^{2} \delta^{2}=v_{3}{ }^{2}, \quad g=\bar{\omega} / \omega_{0}=\bar{S} / S_{g}, \\
& \varphi=\frac{S_{g} v_{3}^{2}}{K \alpha \omega_{0}}, \quad u=\frac{M v_{3}{ }^{2}}{\alpha} v_{s}=N \frac{\rho_{s}}{\rho} v_{s}, \\
& n=\omega_{0} \Delta N, \quad \theta=K \Delta T, \quad \tau=\omega_{0} t .
\end{aligned}
$$

Putting these in, ignoring second-order terms in the perturbations, and substituting Eq. (7) into Eq. (6) we get

$$
\begin{array}{r}
\frac{\partial u}{\partial \tau}-\varphi g \frac{\partial \theta}{\partial x}+\delta^{2} \frac{\partial n}{\partial x}=0, \\
g \frac{\partial n}{\partial \tau}+\frac{\partial \theta}{\partial \tau}+\theta=0, \\
(1-g) \frac{\partial n}{\partial \tau}+\frac{\partial u}{\partial x}-\frac{\partial \theta}{\partial \tau}=0 .
\end{array}
$$

General solutions may be written in the form

$$
\begin{aligned}
& n=n_{0} \sin k x e^{\sigma \tau} \\
& u=u_{0} \cos k x e^{\sigma \tau} \\
& \theta=\theta_{0} \sin k x e^{\sigma \tau} .
\end{aligned}
$$

Solutions with imaginary $\sigma$ correspond to propagating wave modes, real, negative $\sigma$ leads to damping, and any solution with a positive, real part in $\sigma$ means the film is dynamically unstable. Substituting Eqs. (12) into Eqs. (9), (10), and (11), and setting the determinant of the coefficients of the amplitudes $\left(n_{0}, u_{0}\right.$, and $\left.\theta_{0}\right)$ equal to zero, the condition for consistency becomes a cubic equation in $\sigma$,

$$
\sigma^{3}+\sigma^{2}(1-g)+\sigma k^{2}\left(\delta^{2}+\varphi g^{2}\right)+\delta^{2} k^{2}=0
$$

Physically, $g=\bar{S} / S_{g}$ is always much less than 1 . Also, as we shall see below, in cases where these equations apply, $\delta^{2}>\varphi g$, so that if we ignore $g$ relative to 1 we get

$$
(\sigma+1)\left(\sigma^{2}+\delta^{2} k^{2}\right)=0 \text {. }
$$

Thus one solution is damped: $\sigma=-1$ or $\sigma \tau=-\omega_{0} t$. The other solution is $\sigma= \pm i k \delta$, or writing $\sigma \tau=i \omega t$,

$$
\left(\frac{\omega}{k}\right)^{2}=v_{3}{ }^{2}=\frac{\rho_{s}}{\rho} \frac{R T}{M}\left(\frac{\partial \ln P}{\partial \ln N}\right)_{T} .
$$

This solution is a propagating wave with phase velocity $v_{3}$; it is just Atkins' third sound. The difference between Eq. (14) and Eq. (1) will be discussed below. 
For $g \neq 0$ we look for solutions of the form

$$
\sigma=i k \delta+\epsilon
$$

Substituting Eq. (15) into Eq. (13) and retaining terms to first order in $\epsilon$, we get

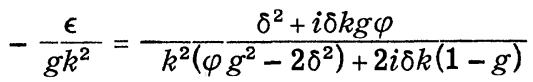

which becomes after rationalizing

$$
\begin{aligned}
-\epsilon / g k^{2}=\{ & \delta^{2} k^{2} \varphi g(2-g)-2 \delta^{4} k^{2} \\
& \left.+i \delta k\left[k^{2} \varphi g\left(\varphi g^{2}-2 \delta^{2}\right)-2 \delta^{2}(1-g)\right]\right\} \\
& \times k^{-2}\left[k^{2}\left(\varphi g^{2}-2 \delta^{2}\right)^{2}+4 \delta^{2}(1-g)^{2}\right]^{-1}
\end{aligned}
$$

Thus there is a hydrodynamic instability if

$$
0<g<2 \delta^{2} /(2-g) \varphi \text {. }
$$

Ignoring $g$ with respect to 2, and substituting in Eqs. (8), we get as the condition for instability,

$$
0<\bar{S}<R \frac{C_{N}}{S_{g}}\left(-\frac{\partial \ln P}{\partial \ln N}\right)_{T} .
$$

The physical origin and significance of this instability will be discussed in Sec. III. ${ }^{13}$

A more general solution for the propagating parts of Eq. (13) with $g \neq 0$ gives a more precise expression for the velocity of third sound

$$
\left(\nu_{3}^{\prime}\right)^{2}=\left[-\frac{\rho}{\rho} \frac{R T}{M}\left(\frac{\partial \ln P}{\partial \ln N}\right)_{T}\right]\left(1-\bar{S} / S_{g}\right)^{-1} .
$$

This should be compared to Atkins' result, ${ }^{1}$

$$
\left(v_{3}^{\prime \prime}\right)^{2}=\left(\rho_{S} / \rho\right) f d(1+T S / L),
$$

where $L$ is the latent heat of the liquid. The terms $T S / L$ and $S / S_{g}$ are small with respect to 1 and we shall ignore them hereafter.

To use Eq. (1) for the interpretation of thirdsound data previous author $\mathrm{s}^{2}, 3$ have written $f d$ $=3 \Delta \mu$, where $\Delta \mu$ is the difference between the chemical potentials of the film and bulk liquid at the same temperature, $\Delta \mu=(R T / M) \ln P_{0} / P$. This comes from making use of the assumption $\mu_{f}=\mu_{g}$ at this point, and in addition assuming $\Delta \mu$ $=($ constant $) / d^{3}$, i. e., that the difference in potential is due to van der Waals attraction to the substrate, and that the van der Waals potential goes inversely as the third power of the distance. There results for the speed of third sound,

$$
\left(v_{3}^{\prime \prime \prime}\right)^{2}=\left(\rho_{s} / \rho\right) 3(R T / M) \ln \left(P_{0} / P\right) .
$$

Equation (22) agrees with Eq. (14) if $3 \ln P_{0} / P$ $=(\partial \ln P / \partial \ln N)_{T}$. Experimentally this indentification is only approximately satisfied. For example, for approximately five layer films at $T \approx 1.5^{\circ} \mathrm{K}^{5}$ $(\partial \ln P / \partial \ln N)=1.9$ when $3 \ln P_{0} / P=1.4$ 。 There are a number of possible reasons for the discrepancy: 1. $\Delta \mu$ is not due only to the van der Waals potential of an atom at the surface; in addition the density of the film differs from that of the liquid, so that the difference in $\mathrm{He}-\mathrm{He}$ interaction between the film and the liquid should enter into $\Delta \mu$.

2. The exponent in the van der Waals potential may not be exactly three. This point has a considerable history of controversy. ${ }^{14}$

3. The internal state of the film may differ from that in the liquid. It is known that $\rho_{S} / \rho$ is always lower in the film than the liquid ${ }^{2} ; \Delta \mu$ may require a term depending on the value of $\rho_{s} / \rho$, in order to insure that if a slab of liquid had a lower than equilibrium value of $\rho_{s} / \rho$ it would be unstable.

In any case it is clear that Eq. (14) is more accurate than Eq. (22) since it involves fewer assumptions and no new ones. Any strange behavior in $\mu_{f}$ has been taken care of in Eq. (14), since adsorption isotherms measure $\mu_{f}$, and Eq. (14) uses only the results of those measurements. It should be noted that Eq. (14) depends on the logarithmic slope of an adsorption isotherm, so that the substrate area, always a problem in adsorption work, need not be known.

Equation (22) has been used to deduce values of $\rho_{s} / \rho$ in the film to be compared to predictions for the average value of the order parameter from microscopic theory. ${ }^{3}$ Whether one uses Eq. (14) or Eq. (22), ad hoc assumptions must be made about whether the first and second layers of film participate in the ordering process.

Equally important, the connection between a microscopically defined quantity such as an order parameter, and the quantity $\rho_{s} / \rho$ that enters the present considerations may be less than obvious. Nevertheless wherever experimental data is available the use of Eq. (14) seems preferable.

\section{INSTABILITY AND THE ONSET CURVE}

In Sec. II we showed that a film described by Eqs. (2), (6), and (7) would be unstable under certain conditions. We shall now discuss the physical nature of the instability, and examine the proposition that it is this instability which leads to the onset curve.

In a crude but helpful picture of a third sound wave, the temperature and thickness oscillations are in opposite phase, the two working against superflow (actually, the phase relation depends on the frequency). Thus at the crest of a wave, the temperature is lower than average due to the excess of superfluid, and outward flow is beginning 
to accelerate. At the trough the film is thin and hot. This situation is depicted by Atkins in Fig. 1 of Ref. (1). Evaporative processes play two conflicting roles. At the crest of the wave there is net condensation, which tends to increase the amplitude of the wave in $N$ (and therefore $T$ ) but this condensation causes warming which tends to decrease the amplitude of $T$ (and therefore $N$ ) waves. At the trough there is evaporation which increases the amplitudes and cooling which decreases them. When the thermal effects dominate, evaporative processes lead only to the attenuation of third sound. But when the mass transfer effect dominates, a perturbation sets a third sound oscillation in motion, and this oscillation grows on each cycle, so that the film is unstable.

One can follow the energy balance in this heuristic picture; indeed, the most convincing way to show that we have correctly described the physics of the instability is to rederive the stability criterion, Eq. (19), from plausibility arguments about the energy balance. Heat flows into the film at low temperature and out at high temperature this is the mechanism of irreversible energy loss and damping. On the other hand, atoms are added at the crest of the wave, fall through the van der Waals field, and evaporate at the trough, feeding energy into the oscillation. The film is unstable when there is a net gain of energy on each cycle in the competition between these two processes.

For simplicity we consider equal intervals of time, $d t$, at the crest and trough of a symmetric wave. Let the amplitudes of the wave be $\delta T$ and $\delta N$, with additional changes due to evaporative processes $d T$ and $d N$. If $d E$ is the energy change, $d Q$ the heat change and $\mu$ the chemical potential, we have due to evaporation at the trough,

$$
\begin{aligned}
d E_{1} & =d Q_{1}+\mu_{1} d N_{1} \\
& =-T_{1} S_{g} K \delta T d t-\mu_{1} K \delta T d t \\
& =-\left(T_{1} S_{g}+\mu_{1}\right) K \delta T d t .
\end{aligned}
$$

Due to condensation at the crest,

$$
d E_{2}=\left(T_{2} S_{g}+\mu_{2}\right) K \delta T d t
$$

Now $T_{1}-T_{2}=2 \delta T$ and $N_{2}-N_{1}=2 \delta N$, so that the net energy change per cycle in the opposite intervals $d t$, becomes,

$$
d E=d E_{1}+d E_{2}=\left[-2 S_{g} \delta T+\left(\mu_{2}-\mu_{1}\right)\right] K \delta T d t
$$

but $\mu_{2}-\mu_{1}=\bar{S}\left(T_{2}-T_{1}\right)-\alpha\left(N_{2}-N_{1}\right)=-2 \bar{S} \delta T-2 \alpha \delta N$, so that

$$
d E=-2\left[\left(S_{g}+\bar{S}\right) \delta T+\alpha \delta N\right] K \delta T d t
$$

We estimate the relationship of the amplitudes of the wave, $\delta N$ and $\delta T$ in the absence of evaporation. From Eq. (6) then,

$$
-\delta N=\left(N C_{N} / T \bar{S}\right) \delta T .
$$

This yields

$$
d E=-2\left[\left(S_{g}+\bar{S}\right)-\left(\alpha N C_{N} / T \bar{S}\right)\right] K(\delta T)^{2} d t
$$

Since $S_{g} \gg \bar{S}$ we get finally $d E>0$ if

$$
\bar{S}<\alpha N C_{N} / T S_{g}
$$

which agrees with Eq. (19). ${ }^{15}$

This does not represent the entire energy balance. Indeed, a gain of energy on each cycle would allow a violation of the second law of thermodynamics. There are other terms in the energy balance of the same order. For example, the evaporation rate constant, $K$, has a rapid temperature dependence, being proportional to the slope of the vapor pressure curve and therefore roughly exponential in temperature. As a result, the waves are not actually symmetric. There is more evaporation at the hot half-cycle than condensation at the cold. The average thickness of the film decreases, providing enough energy to let the waves grow; a rough estimate made by taking $K=K_{0} \pm \delta K$, retaining terms to order $\delta K \delta T$, and using $\delta K / \delta T \approx\left(S_{g} / R T\right) K$ leads to the estimate that the energy lost from the unstable film by this mechanism is greater than that gained so long as $\left(\alpha N C_{N} / \frac{7}{2} S_{g}\right)<T \bar{S}<\left(\alpha N C_{N} / S_{g}\right)$.

We wish to suggest that the disappearance of superflow observed in unsaturated helium films is due to the occurrence of this instability. It is not to be expected that this simple, linear theory should give precise quantitative agreement with the observed onset curve. However, Eqs. (2), (6), and (7) form a model system based on the same set of assumptions used to predict and interpret third sound. This model system has in it an instability which may be taken as a prediction that superflow will cease under certain conditions. Both third sound and the cessation of superflow are observed in real films.

Quantitative comparison with experiment is hindered by the lack of detailed, precise experimental data. However, some estimates may be made. Using existing measurement of $C_{N}{ }^{16}$ and the latent heat of the liquid (to get $S_{g}$ ) and estimating $\partial \ln P / \partial \ln N \approx 3 \ln P_{0} / P$ it is found that the right-hand side of the inequality Eq. (19) does not vary much along the onset curve: it goes from about $0.13 R$ at $1.2^{\circ} \mathrm{K}$ to $\sim 0.15 R$ at $1.5^{\circ}$, to $\sim 0.1 R$ at $1.8^{\circ}$. $S_{g}$ does not change very rapidly, but $C_{N \rightarrow \infty}$ and $(\partial \ln P / \partial \ln N)_{T} \rightarrow 0$ as the bulk 
lambda transition is approached. Sufficiently detailed data for $\bar{S}$ at the onset exists only $T \simeq 1.5^{\circ} .^{5}$ Here it is found that $\bar{S}$ drops abruptly on crossing the onset, from between 0.1 and $0.3 R$ in the superflow region, to a much smaller value where there is no superflow, in excellent agreement with the criterion proposed here.

One of the uncertainties of the model, pointed out in Sec. II, is the assumption needed to get Eq. (5), essentially that if the wave were isothermal there would be no evaporation or condensation. To the extent that this is not correct, the film may be stabilized by extra evaporation at the thick spots and extra condensation at the thin. This would tend to decrease the value of $\bar{S}$ required for instability in a somewhat unpredictable way: The dynamics of the interaction between an oscillating film and a rarified gas (mean free path $\gg$ film thickness) can be very subtle. ${ }^{17,17 a}$ Thus the agreement between theory and experiment at $1.5^{\circ} \mathrm{K}$ may be fortuitous, and one cannot be sure of doing as well at all temperatures. It should be emphasized that even for very rough comparison with the criterion, $\bar{S}$ data of high resolution is needed. The observed behavior at $1.5^{\circ}$ is an inflection in $\int \bar{S} d T$ as the onset is crossed. ${ }^{5}$ It would not be clear from the broad general behavior of $\bar{S}$ in films of that thickness that the criterion is crossed at the onset.

The presence of a free surface and exchange with the gas phase is necessary for the instability. Thus the problem noticed by Brewer et al. ${ }^{6}$ that the film behaves differently from liquid in fine pores of essentially the same dimension, is automatically solved by supposing that the instability causes superflow to stop.

The instability we have described here is spontaneous; it may be set off by a perturbation in any of the film variables, $T, N$, or $v_{s}$. It is furthermore an energetically unfavorable state, as we have argued above, since it involves a decrease in the mean film thickness. It seems likely that the nature of the film to the right of the onset curve in Fig. 1 is not spontaneously unstable, but rather some other state which becomes energetically more stable than the superflow state when the instability occurs in the latter. The first possibility that comes to mind is that the film forms patches, or beads up, since the instability seems to be trying to form such a state. However, there are good arguments against such a state. $\mathrm{He}^{4}$ wets every solid (with the possible exception of solid $\mathrm{He}^{3}$ ) precisely because the $\mathrm{He}-\mathrm{He}$ interaction is much weaker than the interaction of helium with anything else; thus beading up (the opposite of wetting) is a highly unfavorable state for helium. A different Jossibility would be that the over-all film thickness remains essentially constant but the superfluid fraction beads up, i. e., there are local patches of superfluid distributed through a normal fluid background. This would be closely analogous to the intermediate state in superconductivity. Internal stability of the superfluid patches might be achieved by making them of order of the film thickness in lateral dimension (we have made the opposite assumption with regard to wavelengths in deducing a model which is unstable). It would be a very interesting theoretical problem to try to construct possible states and calculate their Gibbs free energy for comparison with the superflow state.

Regardless of the nature of the new state, it cannot be very different energetically from the superflow state in the region close to, but to the left of the onset curve in Fig. 1 (i. e., where superflow is still observed, but close to the onset). Thus regions of this state should be excited from time to time, causing superflow to decay. This would explain the observation that the critical velocity goes continuously to zero at the onset. ${ }^{4}$ In fact we can easily construct a model qualitatively the same as that used to explain the decay of helium supercurrents and the observation of an intrinsic, nongeometry-dependent critical velocity in bulk superfluid close to $T_{\lambda} \cdot{ }^{18}$ We need only assume that, to the left of the onset curve, creation of local patches of the nonsuperflow state lowers the over-all Gibbs energy of the film, but requires that an energy barrier be overcome.

\section{ACKNOWLEDGMENTS}

The author is especially grateful for enthusiastic help from Professor P. G. Saffman and Professor R. P. Feynman. In addition, there have been fruitful discussions with Professors I. Rudnick, D. Bergmann, and R. Elgin.

\footnotetext{
${ }^{1}$ K. R. Atkins, Phys. Rev. 113, 962 (1959); Pollack has considered the effect of normal fluid mobility on the theory of third sound. The effects are small and should be much smaller still for the unsaturated films of interest here, and therefore are not considered in the present work. See G. L. Pollack, Phys. Rev. 143, 103
}

(1966); and 149,72 (1966).

${ }^{2}$ C. W. F. Everitt, K. R. Atkins, and A. Denenstein, Phys. Rev. 136, A1494 (1964).

${ }^{3}$ I. Rudnick, R. S. Kagiwada, J. C. Frazer, and F. F. Guyon, Phys. Rev. Letters 20, 430 (1968); R. S. Kagiwada, J. C. Frazer, I. Rudnick, and D. Bergmann, 
ibid. 22, 338 (1969).

${ }^{4}$ R. P. Henkel, G. Kukichi, and J. D. Reppy, in Proceedings of the Eleventh International Conference on

Low Temperature Physics, to be published.

${ }^{5}$ D. L. Goodstein and R. L. Elgin, Phys. Rev. Letters 22,383 (1969).

${ }^{6}$ D. F. Brewer, A. J. Symonds, and A. L. Thomson, Phys. Rev. Letters 15, 182 (1965).

${ }^{7} \mathrm{D}$. Bergmann, private communication.

${ }^{8}$ See, for example, D. H. Young and A. D. Crowell, Physical Adsorption of Gases (Butterworths, Scientific Publications Ltd., London, 1962), pp. 94, 95.

${ }^{9}$ See E. Long and L. Meyer, Advan. Phys. 2, 1 (1953).

${ }^{10}$ T. L. Hill, J. Chem. Phys. 17, 520 (1949).

${ }^{11}$ This approximation breaks down for low-frequency long-wavelength wave modes, whose period is long compared to whatever relaxation time is associated with the gas at the interface. One can introduce on the right-hand side of Eq. (5) a term proportional to $(\partial P / \partial N) T^{\Delta N}$ to test the importance of the approximation. The ensuing mathematical analysis becomes more complicated, but the qualitative results are unchanged, and the quantitative results presented below, for the velocity of third sound and the criterion for instability, are not changed very much even in the most extreme limit, where the chemical potential of the gas is taken to be constant everywhere. We therefore omit this complication, as did Atkins, in this simple model.

${ }^{12}$ In I it was asserted that thermally activated superflow could be expected to cease if $\bar{S}=0$. It may be seen from the equations of motion that in this model, that is indeed the case. When $\bar{S}=0$ the equations describe waves in $N$ and $v_{s}$, with rapid damping of any temperature perturbation.

${ }^{13}$ It has been suggested (Rudnick, private communication; see also Ref. 3) that $\bar{S}<0$ might represent an unstable region for two fluid hydrodynamics. However, an examination of Eq. (17) shows that the film becomes stable in this region. It is an important result of the present formulation that $\bar{S}$ enters not only into heat flow in the film, in Eq. (6), but also into the two fluid hydrodynamics itself in Eq. (2). Thus if $\bar{S}$ were less than zero, superfluid would flow away from hot spots rather than toward them.

${ }^{14}$ This question is discussed briefly in Ref. 2 .

${ }^{15}$ Along the locus of points $\bar{S}=\alpha N C_{N} / T S_{g}$ evaporative processes have no net effect and the wave propagates undamped. Thus in this region evaporative effects may be considered small, and the use of Eq. (27) is justified.

${ }^{16}$ H. P. R. Frederickse, Physica 15, 860 (1949). The data are reprinted in Ref. 9.

${ }^{17}$ The error introduced depends on the frequency and wavelength of the disturbance, and the relevant corrected criterion would be that for the least stable mode, i.e., the mode in which the present approximation is most nearly correct.

${ }^{17}$ Note added inproof. It has now been shown that if one assumes complete thermal and chemical potential equilibrium across the interface, third sound is always attenuated and the phase velocity is appreciably altered. [D. Bergmann, Report (unpublished)]. Bergmann's assumptions are most valid at low frequencies, whereas the present assumptions are most valid at high frequencies.

${ }^{18}$ J. S. Langer and M. E. Fisher, Phys. Rev. Letters 19 , 560 (1967).

\title{
Bose-Einstein Condensation for a Class of Wave Functions
}

\author{
L. Reatto \\ Istituto di Fisica, Gruppo Nazionale Struttura della Materia, Consiglio Nazionale delle Ricerche, \\ Università di Milano, Milano, Italy \\ (Received 29 January 1969)
}

\begin{abstract}
It is verified that the class of wave functions used in variational calculations for ${ }^{4} \mathrm{He}$ has Bose-Einstein condensation. These trial wave functions have the form of a product of pair functions. Two cases are considered: In the first the correlations in the wave function are assumed to be short range and in the second the correlations include also the long-range part due to the zero-point motion of phonons. The one- and two-dimensional system is briefly considered.
\end{abstract}

The variational method seems to be the only method able to give, from first principles, quantitative information on the ground-state properties of liquid ${ }^{4} \mathrm{He} .{ }^{1}$ It is important to verify that the trial wave 